RUNNING TITLE: Deep sequencing as a tool for TB control

Previously undetected superspreading of Mycobacterium tuberculosis revealed by

\title{
deep sequencing
}

Robyn S. Lee $\mathrm{PhD}^{1,2,3}$, Jean-François Proulx $\mathrm{MD}^{4}$, Fiona McIntosh $\mathrm{BSc}^{5}$, Marcel A. Behr MD William P. Hanage $\mathrm{PhD}^{2,3}$

1. Epidemiology Division, Dalla Lana School of Public Health, University of Toronto, Health Sciences Building, 155 College Street, Room HS586, Toronto, ON, Canada, M5T 3M7

2. Center for Communicable Disease Dynamics, Harvard T. H. Chan School of Public Health, 677 Huntington Avenue, Kresge Building, Room 506I, Boston, MA, USA, 02115

3. Department of Epidemiology, Harvard T. H. Chan School of Public Health, Boston, MA, USA, 677 Huntington Avenue, Kresge Building, $5^{\text {th }}$ floor, Boston, MA, USA, 02115

4. Nunavik Regional Board of Health and Social Services, Kuujjuaq, Québec, Canada

5. The Research Institute of McGill University Health Centre, Montréal, Québec, Canada

*Address correspondence to:

17 Dr. Robyn Lee, PhD

18 Epidemiology Division

19 Dalla Lana School of Public Health, University of Toronto

20 Health Sciences Building

$21 \quad 155$ College Street, Room HS 586

22 Toronto, Ontario, Canada

23 M5T 3M7

24 Email: robyn.s.c.lee@gmail.com

25 Word count: 3729

26 Figures: 1

27 Tables: 1 
RUNNING TITLE: Deep sequencing as a tool for TB control

30 Abstract - 150/150 words

31 Tuberculosis disproportionately affects the Canadian Inuit. To address this, it is imperative we

32 understand transmission dynamics in this population. We investigate whether 'deep' sequencing

33 can provide additional resolution compared to standard sequencing, using a well-characterized

34 outbreak from the Arctic (2011-2012, 50 cases). Samples were sequenced to 500-1000x and

35 reads were aligned to a novel local reference genome generated with PacBio SMRT sequencing.

36 Consensus and heterogeneous variants were identified and compared across genomes. In contrast

37 with previous genomic analyses using 50x depth, deep sequencing allowed us to identify a

38 novel super-spreader who likely transmitted to up to 17 other cases during the outbreak (35\% of

39 all cases that year). It is increasingly evident that within-host diversity should be incorporated

40 into transmission analyses; deep sequencing can facilitate accurately detection of super-spreaders

41 and corresponding transmission clusters. This has implications not only for TB, but all genomic

42 studies of transmission - regardless of pathogen. 
RUNNING TITLE: Deep sequencing as a tool for TB control

\section{Introduction.}

44 Tuberculosis (TB) in Canada is highest among the Inuit, an Indigenous population with a rate

45 over 300x that of the non-Indigenous Canadian-born population in 2016. ${ }^{1}$ Canada recently set a

46 goal of TB elimination in the Inuit by $2030,{ }^{1}$ which will not be achieved without halting ongoing

47 transmission. Previous studies have used genomic data either alone or in conjunction with

48 classical epidemiology to investigate TB transmission dynamics in the Canadian North, ${ }^{2-4}$ with

49 the aim of identifying clusters to help guide public health interventions. Thus far, such studies

50 have relied on identifying consensus single nucleotide polymorphisms (cSNPs), consistent with

51 prevailing methodology in this field.

53 Recent studies suggest that incorporation of within-host diversity into genomic analyses may

54 provide greater resolution of transmission than cSNP-based approaches alone. ${ }^{5-8}$ This may be

55 particularly important for investigation of outbreaks occurring over short time scales and/or in

56 settings such as the Canadian North, where the genetic diversity of circulating strains is

57 especially low. In both of these circumstances, it is common to find many samples separated by

58 zero cSNPs, hindering accurate source ascertainment. To investigate this hypothesis, we used

59 deep sequencing (i.e., to $\sim 10$-fold more than standard, or 500-1000x) to re-evaluate transmission

60 in a densely-sampled outbreak in Nunavik, Québec.

61

62 This outbreak, which has been previously described, ${ }^{4,9}$ comprised 50 microbiologically-

63 confirmed cases of TB who were diagnosed in a single Inuit community between 2011-2012 - a

64 rate of 5,359/100,000 for that year. Genomic epidemiology analyses using sequencing depths of

$65 \sim 50 \mathrm{x}$ that are standard in such work, identified multiple clusters of transmission in this outbreak, 


\section{RUNNING TITLE: Deep sequencing as a tool for TB control}

$66{ }^{4}$ however, there was insufficient genetic variation detected to infer precise person-to-person

67 transmission events within these subgroups, given the short time frame and low mutation rate of

68 M. tuberculosis ( 0.5 SNPs/genome/year for Lineage $\left.4^{10}\right)$. In this study, we illustrate how

69 within-host diversity can be incorporated into transmission analyses and in doing so, find new

70 features of the transmission networks in this community, in particular, identifying a previously

71 unrecognized superspreading event. We highlight a potential role for deep sequencing in public

72 health investigations, with implications for TB control in Canada's North as well as other high-

73 transmission environments.

\section{Materials and methods.}

76 Study subjects. All 50 samples from the 2011-2012 outbreak ${ }^{4}$ were eligible for inclusion, as

77 well as samples from all cases $(n=15)$ diagnosed in same village in the preceding five years

78 (2007 onwards), 13/15 of which were caused by the same strain of M. tuberculosis (the 'Major

$79 \quad[\mathrm{Mj}]-\mathrm{III}$ ' sublineage ${ }^{3}$ ). There were two episodes of recurrent TB (i.e., where an individual had

80 microbiologically-confirmed TB once, was cured, but developed TB again during the study

81 period); otherwise, all samples are from unique individuals. All cases had pulmonary TB that

82 was Lineage 4 (Euro-American ${ }^{4}$ ). Cross-contamination was ruled out as described in ${ }^{4}$.

84 DNA extraction and sequencing. Laboratory methods are described in detail in the

85 Supplementary Material. The Illumina HiSeq 4000 was used for paired-end 100bp sequencing.

86 To obtain the target depth of coverage, pooled libraries were run on four independent lanes. 


\section{RUNNING TITLE: Deep sequencing as a tool for TB control}

Bioinformatics. Quality control of genomic data is described in detail in the Supplemental

89 Material. Reads were aligned using the Burrows Wheeler Aligner MEM algorithm (v.0.7.15 ${ }^{11}$ )

to the H37Rv reference (NC_000962.3 in the National Center for Biotechnology Information

$91[\mathrm{NCBI}]$ RefSeq database) and sorted using Samtools (v.1.5 ${ }^{12}$ ). Analyses were later repeated

92 using a local reference genome (described below). Reads with ambiguous mapping were

93 excluded, as were reads with excessive soft-clipping (i.e., more than $20 \%$ of read length) based

94 on our previous work. ${ }^{6}$ Duplicate reads were marked using Picard MarkDuplicates (v.2.9.0,

95 https://broadinstitute.github.io/picard/) and reads were locally re-aligned around indels using

96 Genome Analysis ToolKit (GATK, v. $3.8^{13}$ ). All sites were called using GATK's Unified

97 Genotyper algorithm.

99 Variants were filtered for quality using custom Python scripts (v.3.6) with the following

100 thresholds: Phred < 50, Root Mean Squared Mapping Quality (RMS-MQ) $\leq 30$, depth (DP) $<20$,

101 Fisher Strand Bias (FS) $\geq 60$ and read position strand bias (ReadPos) $<-8 .{ }^{6}$ cSNPs were

102 classified as positions where $\geq 95 \%$ of reads were the alternative allele (ALT), hSNPs were

103 classified as positions where $>5 \%$ and $<95 \%$ of reads were ALT, and positions with the ALT

104 present in $<5 \%$ of reads were classified as 'reference'. We also compared inferences of

105 transmission from this analysis to i) when these thresholds were increased to the minimum

106 values among cSNPs in the initial H37Rv analysis, and ii) when cSNPs were classified using a

107 threshold of $\geq 99 \%$, and hSNPs were classified when $1 \%<$ ALT $<99 \%$, in order to assess the

108 robustness of inferences to different filtering protocols. 
RUNNING TITLE: Deep sequencing as a tool for TB control

110 Low-quality variants, variants in proline-proline-glutamic acid (PE) and proline-glutamic-

111 acid/polymorphic-guanine-cytosine-rich sequence (PE_PGRS) genes, transposons, phage and

112 integrase, and positions with missing data, were excluded. All samples were drug-susceptible,

113 except for MT-6429, which was rendered resistant to isoniazid by a frameshift deletion at

114 position 1284 in the catalase-peroxidase gene $k a t G$. As such, positions associated with drug

115 resistance were not masked in this analysis.

117 Concatenated cSNP alignments were generated excluding positions with hSNPs. Pairwise cSNP

118 distances between samples were computed using snp-dists (v.0.6, available at

119 https://github.com/tseemann/snp-dists). The frequency of hSNPs at each position in the genome

120 was tabulated and hSNPs were reviewed to identify variants shared between samples.

122 Phylogenetics and clustering. Core cSNP alignments were used to generate maximum

123 likelihood trees using IQ-Tree (v.1.6.8 ${ }^{14}$ ). Model selection was based on the lowest Bayesian

124 Information Criterion. Hierarchical Bayesian Analysis of Population Structure ${ }^{15}$ was run in R

125 (v.3.5.2) to identify clusters. See the Supplementary Material for additional detail.

127 Single Molecule Real-Time (SMRT) sequencing and assembly. To examine the influence of

128 potential alignment errors in identification of hSNPs, we used SMRT sequencing with the

129 PacBio RSII platform to create a local reference genome for the outbreak. Sample MT-0080 was

130 chosen for sequencing because this was previously identified as the probable source for as many

131 as 19 of the 50 cases diagnosed in 2011-2012. ${ }^{4}$ A single colony from the culture was selected for 
RUNNING TITLE: Deep sequencing as a tool for TB control

132 SMRT sequencing and Illumina MiSeq (for polishing of the long-read assembly). Further detail

133 is provided in the Supplementary Material.

135 Long-reads were assembled and corrected using Canu (v.1.7.1 $\left.{ }^{16}\right)$. Pilon (v.1.23 ${ }^{17}$ ) was then used 136 to polish the assembly and was re-run until no further corrections were possible. Quast (v.5.0.2,

$13{ }^{18}$ ) was used to evaluate assembly quality. RASTtk (v.2.0 ${ }^{19}$ ) was used for annotation, to identify 138 regions for masking as previous.

140 Epidemiological data. Epidemiological and clinical data were collected on all cases and contacts

141 using standardized questionnaires, as part of the routine public health response.

143 Statistical analyses. A two-sample test of proportions was used to compare overall proportions

144 across references, and the Wilcoxon Signed Rank test was used to compare paired SNP

145 distances. Analyses were done in Stata (v.15, StataCorp, College Station, TX, USA).

147 Data availability. Sequencing data and the assembly for MT-0080 are available on the NCBI's

148 Sequence Read Archive under BioProject PRJNA549270.

150 Ethics. Ethics approval was obtained from the Institutional Review Board (IRB) of the Harvard

151 T.H. Chan School of Public Health (IRB18-0552) and the IRB of McGill University Faculty of

152 Medicine (IRB A02-M08-18A). All data was analyzed in non-nominal fashion. This study was

153 done with approval of and in collaboration with the Nunavik Regional Board of Health and

154 Social Services. 
RUNNING TITLE: Deep sequencing as a tool for TB control

156 Results.

157 62/65 (95.4\%) available TB samples from cases diagnosed between 2007-2012 were successfully

158 sequenced and passed quality control. This included 48/49 (98.0\%) of the samples with an

159 identical Mycobacterial Interspersed Repetitive Units Variable Number Tandem Repeats

160 (MIRU-VNTR) pattern during the outbreak year. The remaining three samples could not be re-

161 grown. Reads that were non-MTBC were removed (Table S1) and there was no obvious

162 association between percent contamination and hSNP frequency. Epidemiological and clinical

163 data on all outbreak cases are described in ${ }^{4}$.

165 Average genome coverage and depth across the H37Rv reference was 98.64\% [SD 0.07\%] and

167 hSNPs across all individual samples (Table S2). Excluding positions that were invariant

168 compared to the reference or where any sample was missing and/or was low-quality resulted in a

169 core alignment of $860 \mathrm{cSNP}$ positions and $136 \mathrm{hSNP}$ positions (note, these are not mutually

170 exclusive, as positions with cSNPs in some samples may have hSNPs in others).

17242 positions had hSNPs that were shared across all 62 samples (Table 1, Supplementary

173 Dataset 1A). Depth of coverage at these positions was, on average, 39\% higher than the average

174 depth across the same sample (SD 36.7\%, Supplementary Dataset 1B). Along with manual

175 review of alignments (Figure S1), this suggested that many of these were false positives,

176 potentially due to alignment error (e.g., from underlying structural variation in our samples

177 compared to the H37Rv reference). 
RUNNING TITLE: Deep sequencing as a tool for TB control

179 To address this, we generated a local reference genome for the outbreak, MT-0080_PB. Quality metrics for the MT-0080_PB assembly are given in Table S3. Compared to H37Rv, mean

181 genome coverage and depth were higher with MT-0080_PB (at 99.33\% [SD 0.09\%] and 717.07

182 [SD 93.01], respectively), fewer positions were missing/low-quality ( $\mathrm{p}<0 \cdot 00005$, Table 1), and

183 overall, fewer variable positions were detected $(\mathrm{p}<0.00005)$. While core cSNP distances were

184 similar between samples regardless of the reference (Table 1), the number of hSNPs was greatly

185 reduced using MT-0080_PB (Table S2); while 4,897 hSNPs were identified across all individual

186 samples using H37Rv, only 125 hSNPs were identified using MT-0080_PB. There were also no

187 hSNPs shared across all 62 samples using MT-0080_PB. Together, these findings support our

188 hypothesis that alignment error is responsible for many of the detected variants, and indicate a

189 local reference is important for accurate identification of hSNPs. All further results presented are

190 based on the MT-0080_PB alignment.

191

192 A maximum likelihood tree was generated from 94 core cSNP positions (excluding sites

193 invariant across all samples and the reference) compared to MT-0080 (Figure 1A). Consistent

194 with previous work, ${ }^{4}$ hierBAPS identified two main sub-lineages ('Mj-V' and 'Mj-III' per $^{3}$ ),

195 with three sub-clusters $(\mathrm{Mj}-\mathrm{IIIA} / \mathrm{B} / \mathrm{C})$.

197 hSNPs identify super-spreaders and more accurately resolve transmission clusters. The core

198 cSNPs and hSNPs between samples are shown in Supplemental Dataset 2A, with the sub-

199 groups identified in the original analysis indicated. Overlaying hSNPs with the cSNP-based

200 analysis revealed a novel super-spreader (MT-504) in Cluster Mj-IIIB, undetected by genomic 


\section{RUNNING TITLE: Deep sequencing as a tool for TB control}

201 epidemiology analyses relying on lower sequencing depth. ${ }^{4}$ MT-504 had smear-positive cavitary

202 disease and was diagnosed in late 2011; previous analyses had found this case shared a single

203 cSNP with four other cases diagnosed from March - December of 2012 (position 276,685

204 according to H37Rv / 276,544 in the MT-0080_PB alignment, Supplementary Dataset 2).

205 Coupled with epidemiological data on contact (shared attendance at local community 'gathering

206 houses', social venues specifically identified by public health during the outbreak), this strongly

207 supported transmission from MT-504 to other members of this subgroup. In contrast, the other

208 subgroup of $\mathrm{Mj}$-IIIB with 13 cases did not share this cSNP. This initially refuted transmission, as

209 we would expect 0 SNPs to accrue in recent transmission given the short time period, low

210 mutation rate of TB, and overall low diversity of strains circulating in the village (Figure 1B).

211 Instead, we previously postulated that the first smear-positive case in this subgroup (MT-2474,

212 diagnosed in May 2012) led to the majority of transmission (note, the first smear-negative case in

213 this subgroup was diagnosed in March 2012). However, deep sequencing data suggest otherwise;

214 these data show that MT-504 harboured both the reference (133 reads [19.1\%]) allele, present in

215 the subgroup of 13, as well as the alternative allele (563 reads [80.9\%]) at this position (Figure

216 1C). As MT-504 was the first contagious case diagnosed in Mj-IIIB, and all 13 cases in this

217 subgroup had attended or resided in a gathering house (with 9/13 [69.2\%] reporting attendance at

218 the same houses as MT-504), this strongly suggests that MT-504 is in fact the most probable

219 source for both subgroups.

221 hSNP analysis adds support for suspected transmission. Sample 68995 and MT-5543 were

222 from 2007, and were the only strains from the Mj-VA sub-lineage in this village. Previous

223 analysis indicated $\mathrm{Mj}-\mathrm{VA}$ strains from other villages were distantly related, ${ }^{4}$ while these two 
RUNNING TITLE: Deep sequencing as a tool for TB control

samples were separated from one another by zero core cSNPs. This suggests direct transmission

225 between these historical cases, a hypothesis strongly supported by hSNP analysis, as the samples

226 share hSNPs that are not found in any other sample in the dataset. These hSNPs were present

227 even when highly conservative filtering thresholds were used (Supplemental Dataset 2B).

228 Importantly, these hSNPs were not detected when using H37Rv as the reference.

Potential utility for discriminating TB recurrence. Six individuals had TB recurrence in 2011-

MT-1838 in 2012; Patient 2: samples MT-5543 in 2007 and MT-1206 in 2012, Figure 1A). strain, rather than relapse with the strain causing their original disease. Investigation of withinhost diversity strongly supported this conclusion; using deep sequencing, we verified that there was a single, different strain present at both baseline and their second episodes of TB. There was no evidence for mixed infection at either baseline or second episode with these strains, more definitively ruling out relapse in this low diversity setting (Supplemental Dataset 2A/B/C).

241 variants using the prior cSNP/hSNP thresholds, we re-ran our analysis such that hSNPs were

242 classified when 1\% < ALT < 99\%. Quality scores for individual cSNPs and hSNPs are given in

243 Table S4 and the core cSNP/hSNP alignment is shown in Supplemental Dataset 2C. While our

244 primary analysis using a threshold of $>95 \%$ for cSNPs identified a single cSNP $(A>G)$ shared

245 across all samples compared to MT-0080_PB, close examination of the MT-0080 deep

246 sequencing data (obtained using DNA from a sweep of the plate) showed that this sample had 
RUNNING TITLE: Deep sequencing as a tool for TB control

247 both alleles at this position, with only the minority ' $A$ ' allele (33 reads/1189 [2.8\%]) isolated for

248 SMRT sequencing. Based on this, we recommend sequencing samples both using a clean sweep

249 (with an alternative sequencing platform) and a single colony pick when generating a reference

250 genome for TB, as using the latter alone may introduce error and affect epidemiological

251 inferences. With this exception, no other informative hSNPs were detected using these

252 thresholds.

254 Discussion.

255 As the TB epidemic continues among the Canadian Inuit, targeted public health interventions are

256 essential to halt ongoing transmission. In order to do so, it is important that transmission events

257 and associated risk factors are accurately identified. Our previous work suggested that hSNP

258 analysis could enhance resolution of TB transmission ${ }^{6}$. To investigate how this approach could

259 be applied for TB control, we used deep sequencing to re-examine a major TB outbreak in the

260 Canadian Arctic.

262 Several recent studies, including work by the authors ${ }^{6}$, have shown that M. tuberculosis within-

263 host diversity can be transmitted between individuals ${ }^{8,20}$. Using deep sequencing data allowed us

264 to better identify this diversity in a Nunavik outbreak compared to previous analyses with

265 standard sequencing depth, ${ }^{3,4}$ and facilitated detection of a novel super-spreader who was likely

266 responsible for $\sim 1 / 3$ of the cases from 2011-2012. Super-spreading has been described in a

267 number of pathogens, ${ }^{21}$ including TB. ${ }^{22}$ Our findings suggest this can play an important role in

268 driving TB outbreaks. We therefore propose that investigation of within-host diversity is

269 necessary to ensure detection of such super-spreaders in this context, and potentially other high- 


\section{RUNNING TITLE: Deep sequencing as a tool for TB control}

270 transmission environments as well, in order to accurately identify transmission networks and

271 associated risk factors. We typically only know that a person is a super-spreader retrospectively,

272 i.e., once they have already transmitted to many others and once corresponding genomic data is

273 available. However, if we can identify the characteristics associated with this phenomenon at the

274 population level, this could be used prospectively to predict whether cases are likely to be super-

275 spreaders - as they are diagnosed. This could allow resources to be better allocated, for example,

276 investigation of their contacts could be prioritized and/or targeted screening of the social venues

277 they attend could be rapidly initiated, potentially leading to faster detection of secondary cases

278 and initiation of prophylaxis for new infections. In the case of MT-504, nearly all of the

279 secondary cases had attended the same local community gathering houses as the putative source;

280 this also strongly suggests the importance of these venues in facilitating transmission in this

281 setting.

283 Several studies have used genomics to investigate TB recurrence, ${ }^{23-25}$ however, the methods used

284 to assess for mixed infection at either time point have been inconsistent and may not be sufficient

285 to discriminate recurrence in settings with low strain diversity. In this analysis, we provide proof-

286 of-principle that deep sequencing can potentially help rule out relapse. The distinction between

287 relapse and re-infection is important at individual and population levels; high rates of relapse in a

288 community would indicate a problem with treatment or adherence, potentially warranting

289 changes to clinical management, while re-infection would indicate the need for public health

290 interventions such as activate case finding. Also, individuals in Nunavik who have had prior

291 treatment for active TB disease in the past are also not routinely offered prophylaxis on re-

292 exposure, based on historical data suggesting $\sim 80 \%$ protection is afforded by prior infection. ${ }^{26}$ 


\section{RUNNING TITLE: Deep sequencing as a tool for TB control}

293 The degree to which re-infection drives recurrence in Nunavik is currently unknown, but if re-

294 infection is the primary cause, this clinical practice may need to be re-evaluated. Population-

295 level genomic studies are currently underway to evaluate this.

297 To use deep sequencing to investigate within-host diversity, it is critical we minimize false

298 positive hSNPs. We have shown that using a local strain as a reference not only reduces error,

299 but improves detection of epidemiologically-informative variants. Genomic differences between

300 outbreak strains and H37Rv have been previously illustrated by ${ }^{27,28}$, with O'Toole et al. ${ }^{28}$

301 warning that clinical TB strains may be needed to fully detect virulence genes in reference-based

302 analyses. We propose these are also warranted for hSNP analysis. Where possible, we

303 recommend using long-read sequencing to generate complete and local reference genomes.

305 Overall, our study has a number of strengths. Firstly, we had access to a densely-sampled

306 outbreak, which was previously investigated using 'standard' sequencing depth and for which

307 detailed epidemiological data was available. This allowed us to readily compare methodological

308 approaches, showing how and when deep sequencing might be beneficial for public health. In

309 doing this, we have identified important methodological considerations for hSNP detection, with

310 implications for transmission analyses, but also potentially for resistance prediction as well. ${ }^{29}$

311 Finally, the use of long-read data has allowed us to completely assemble a novel TB genome

312 from Nunavik. This will serve as a valuable resource for future studies of transmission in

313 Nunavik (given the low strain diversity in the region ${ }^{3}$ ), as well as other Inuit territories. 


\section{RUNNING TITLE: Deep sequencing as a tool for TB control}

315 A potential limitation of this work is that, given the historical nature of the outbreak, deep

316 sequencing was done using DNA extracted from culture. Due to methodological challenges of

317 sequencing directly from sputum, ${ }^{30-32}$ few studies have examined the effect of culture on genome

318 diversity. A recent study by Shockey et $a l^{33}$. using cSNPs suggests that variation may be lost

319 during the culturing process, however authors did not examine the impact on hSNPs or

320 transmission, and several other studies ${ }^{31,32,34}$ previously found congruent results between cSNP

321 analyses from culture versus raw samples. In terms of hSNPs, Votintseva et al. found no

322 difference in the number detected between approaches. ${ }^{31}$ While ${ }^{32,34}$ reported detecting fewer

323 hSNPs with sequencing from culture versus from sputum, in ${ }^{34}$, the median hSNPs was only 4.5

324 versus 5 hSNPs, respectively - a difference that may not be clinically significant, regardless of

325 statistical significance. Given the inconsistency of results and paucity of data, further study is

326 needed to understand how hSNP diversity may be affected by the culturing process, and to assess

327 whether this affects inferences of transmission. We note that it is likely that enhanced detection

328 of the hSNPs present in sputum would improve the resolution over that which we present in this

329 work.

331 Another potential limitation is that, while we can compare the epidemiological inferences made

332 between our previous analysis and our deep sequencing analysis, the bioinformatics pipelines

333 themselves are not directly comparable. Methods to accurately identify hSNPs and incorporate

334 them into transmission analyses are currently an area of active research. We illustrated in our

335 recent paper ${ }^{6}$ that additional steps and strict thresholds must be used to minimize false positive

336 hSNPs, and have conducted the current analysis in consideration of this. However, we note that

337 pre-filtering, our 2015 analysis found that MT-504 had five reference alleles at position 276,685 


\section{RUNNING TITLE: Deep sequencing as a tool for TB control}

338 in the H37Rv alignment (out of 75) and randomly downsampling the current data to simulate

$339 \sim 50 \mathrm{x}$ yielded similar results (5/47 reads at position 276,544 aligned to MT-0080_PB). As most

340 genomic studies of TB employ conservative thresholds of 75-90\% allele frequency to classify

341 cSNPs, many bioinformatics pipelines would consider this heterogeneity as potentially suspect at

342 standard sequencing depth. This therefore reinforces the need for greater depth and/or analytic

343 approaches (e.g., ${ }^{35}$ ) to ensure accurate discrimination of sequencing/analytic error from true

344 variation.

346 In summary, we have found evidence of mixed variants with important epidemiologic

347 implications that would not have been detected with standard methods and common filtering

348 criteria. This illustrates that genomic methods, while powerful, still require careful interpretation

349 and can still harbor considerable ambiguity when comparing very close links in a transmission

350 chain - a finding whose relevance likely extends far beyond TB, given the increasing number of

351 pathogens undergoing genomic investigation. We demonstrate that deep sequencing can aid in

352 transmission analyses, in particular by allowing accurate identification of TB super-spreaders

353 and key associated epidemiological characteristics. In terms of TB control, this work has

354 important implications for the Canadian North as well as other regions with high TB

355 transmission; as next-generation sequencing becomes a mainstay in public health surveillance, it

356 is critical we recognize the limitations of analyses done using routine sequencing data and

357 consider where and when deep sequencing might be warranted. Although costs continue to

358 decline, we recognize deep sequencing of all samples in an outbreak may not be economically

359 viable for every public health unit. As such, we propose that public health units using routine

360 sequencing for tuberculosis consider - at a minimum - targeted deep sequencing of the more 
RUNNING TITLE: Deep sequencing as a tool for TB control

361 contagious (e.g., smear-positive) cases, in lieu of all samples, to help ensure accurate

362 identification of super-spreaders and clusters of transmission. This may help TB control

363 programmes better understand the risk factors for such transmission and enable prioritization of

364 public health resources in future outbreaks.

367 Acknowledgements. We would like to thank the council of the village for their ongoing support

368 of this work. We also acknowledge staff from the Centre Locales des Services Communautaires

369 and the Nunavik Regional Board of Health and Social Services for their hard work during the

370 outbreak. We would like to thank Dr. Hafid Soualhine (currently at the National Reference

371 Centre for Mycobacteriology in the National Microbiology Laboratory, Public Health Agency of

372 Canada) for previous confirmation of the frameshift deletion in katG of MT-6429. We also thank

373 Dr. Anders Gonçalves da Silva and Dr. Glen Carter of the Microbiological Diagnostic Unit

374 Public Health Laboratory at the University of Melbourne for their helpful input on high-quality

375 SMRT sequencing. Library preparation and sequencing for all samples was done at the Genome

376 Québec / McGill Innovation Centre and high-performance computing was done using the

377 Odyssey cluster from the Faculty of Arts and Science, Harvard University. This work was

378 supported by an R01 grant from the National Institutes of Health, awarded to WPH

379 (R01AI128344). RSL is also supported by a Fellowship from the Canadian Institutes of Health

380 Research (MFE 152448). MAB holds a Canadian Institutes of Health Research Foundation Grant

381 (FDN-148362).

382

383 Competing interests. Authors have no competing interests to declare. 
RUNNING TITLE: Deep sequencing as a tool for TB control

385 Contributions. RSL and WPH conceived and designed the study. RSL designed and did the

386 analyses, made the tables and figures, interpreted the data, and wrote the first draft of

387 manuscript. JFP provided epidemiological data. MAB provided the bacterial samples, as well as

388 laboratory reagents, Biosafety Level 3 access and technician labour in-kind to RSL. FM did the

389 culture and DNA extraction for the HiSeq and PacBio SMRT sequencing. WPH reviewed the

390 initial draft. All authors provided feedback and reviewed the final version of the manuscript

391 before submission. RSL had full access to the data and final responsibility for the decision to

392 submit for publication. 
RUNNING TITLE: Deep sequencing as a tool for TB control

\section{References.}

$397 \quad$ 1. Kanatami IT. Inuit Tuberculosis Elimination Framework. 2018.

398 2. Tyler AD, Randell E, Baikie M, et al. Application of whole genome sequence analysis to 399 the study of Mycobacterium tuberculosis in Nunavut, Canada. PLoS One 2017; 12(10):

$400 \mathrm{e} 0185656$.

401 3. Lee RS, Radomski N, Proulx JF, et al. Population genomics of Mycobacterium 402 tuberculosis in the Inuit. Proc Natl Acad Sci U S A 2015; 112(44): 13609-14.

403 4. Lee RS, Radomski N, Proulx JF, et al. Reemergence and amplification of tuberculosis in 404 the Canadian arctic. J Infect Dis 2015; 211(12): 1905-14.

$405 \quad 5 . \quad$ Worby CJ, Lipsitch M, Hanage WP. Shared Genomic Variants: Identification of 406 Transmission Routes Using Pathogen Deep-Sequence Data. Am J Epidemiol 2017; 186(10): 407 1209-16.

408 6. Martin MA, Lee RS, Cowley LA, Gardy JL, Hanage WP. Within-host Mycobacterium 409 tuberculosis diversity and its utility for inferences of transmission. Microb Genom 2018; 4(10).

4107 7. Meehan CJ, Goig GA, Kohl TA, et al. Whole genome sequencing of Mycobacterium 411 tuberculosis: current standards and open issues. Nature Reviews Microbiology 2019.

412 8. Seraphin MN, Norman A, Rasmussen EM, et al. Direct transmission of within-host 413 Mycobacterium tuberculosis diversity to secondary cases can lead to variable between-host 414 heterogeneity without de novo mutation: A genomic investigation. EBioMedicine 2019; 47: 293415300.

$4169 . \quad$ Lee RS, Proulx JF, Menzies D, Behr MA. Progression to tuberculosis disease increases 417 with multiple exposures. Eur Respir J 2016; 48(6): 1682-9.

418 10. Menardo F, Duchene S, Brites D, Gagneux S. The molecular clock of Mycobacterium 419 tuberculosis. PLoS Pathog 2019; 15(9): e1008067.

$420 \quad 11$ Li H. Aligning sequence reads, clone sequences and assembly contigs with BWA-MEM. 421 arXiv 2013: 1303.3997v2.

422 12. Li H, Handsaker B, Wysoker A, et al. The Sequence Alignment/Map format and 423 SAMtools. Bioinformatics 2009; 25(16): 2078-9.

424 13. McKenna A, Hanna M, Banks E, et al. The Genome Analysis Toolkit: a MapReduce framework for analyzing next-generation DNA sequencing data. Genome Res 2010; 20(9): 1297303.

14. Nguyen LT, Schmidt HA, von Haeseler A, Minh BQ. IQ-TREE: a fast and effective stochastic algorithm for estimating maximum-likelihood phylogenies. Mol Biol Evol 2015; 32(1): 268-74.

15. Cheng L, Connor TR, Siren J, Aanensen DM, Corander J. Hierarchical and spatially explicit clustering of DNA sequences with BAPS software. Mol Biol Evol 2013; 30(5): 1224-8. 16. Koren S, Walenz BP, Berlin K, Miller JR, Bergman NH, Phillippy AM. Canu: scalable and accurate long-read assembly via adaptive k-mer weighting and repeat separation. Genome Res 2017; 27(5): 722-36.

435 17. Walker BJ, Abeel T, Shea T, et al. Pilon: an integrated tool for comprehensive microbial 436 variant detection and genome assembly improvement. PLoS One 2014; 9(11): e112963.

437 18. Gurevich A, Saveliev V, Vyahhi N, Tesler G. QUAST: quality assessment tool for genome 438 assemblies. Bioinformatics 2013; 29(8): 1072-5. 
RUNNING TITLE: Deep sequencing as a tool for TB control

19. Brettin T, Davis JJ, Disz T, et al. RASTtk: a modular and extensible implementation of the RAST algorithm for building custom annotation pipelines and annotating batches of genomes. Sci Rep 2015; 5: 8365.

20. Guthrie JL, Strudwick L, Roberts B, et al. Whole genome sequencing for improved understanding of Mycobacterium tuberculosis transmission in a remote circumpolar region. Epidemiology and Infection 2019; 147.

21. Stein RA. Super-spreaders in infectious diseases. Int J Infect Dis 2011; 15(8): e510-3. 22. Kline SE, Hedemark, L.L., Davies, S.F. Outbreak of tuberculosis among regular patrons of a neighborhood bar. New Engl J Med 1995; 333(4): 222-7.

23. Witney $A A$, Bateson AL, Jindani $A$, et al. Use of whole-genome sequencing to distinguish relapse from reinfection in a completed tuberculosis clinical trial. BMC Med 2017; 15(1): 71. 24. Bryant JM, Harris SR, Parkhill J, et al. Whole-genome sequencing to establish relapse or re-infection with Mycobacterium tuberculosis: a retrospective observational study. The Lancet Respiratory Medicine 2013; 1(10): 786-92.

25. Guerra-Assuncao JA, Houben RM, Crampin AC, et al. Recurrence due to relapse or reinfection with Mycobacterium tuberculosis: a whole-genome sequencing approach in a large, population-based cohort with a high HIV infection prevalence and active follow-up. $J$ Infect Dis 2015; 211(7): 1154-63.

26. Menzies D. Issues in the management of contacts of patients with active pulmonary tuberculosis. Can J Public Health 1997; 3: 197-201.

27. Roetzer A, Diel R, Kohl TA, et al. Whole genome sequencing versus traditional genotyping for investigation of a Mycobacterium tuberculosis outbreak: a longitudinal molecular epidemiological study. PLoS Med 2013; 10(2): e1001387.

28. O'Toole RF, Gautam SS. Limitations of the Mycobacterium tuberculosis reference genome H37Rv in the detection of virulence-related loci. Genomics 2017; 109(5-6): 471-4. 29. Liu Q, Via LE, Luo T, et al. Within patient microevolution of Mycobacterium tuberculosis correlates with heterogeneous responses to treatment. Sci Rep 2015; 5: 17507.

30. Brown AC, Bryant JM, Einer-Jensen K, et al. Rapid Whole-Genome Sequencing of Mycobacterium tuberculosis Isolates Directly from Clinical Samples. J Clin Microbiol 2015; 53(7): 2230-7.

31. Votintseva AA, Bradley, P., Pankhurst, L., del Ojo Elias, C., Loose, M., Nilgiriwala, K., Chatterjee, A., Smith, E.G., Sanderson, N., Walker, T.M., Morgan, M.R., Wyllie, D.H., Walker, A.S., Peto, T.E.A., Crook, D.W., Iqbal, Z. Same-day diagnostic and surveillance data for tuberculosis via whole genome sequencing of direct respiratory samples. J Clin Microbiol 2017. 32. Doyle RM, Burgess, C., Williams, R., Gorton, R., Booth, H., Brown, J., Bryant, J.M., Chan, J., Creer, D., Holdstock, J., Kunst, H., Lozewicz, S., Platt, G., Yara Romero, E., Speight, G., Tiberi, S., Abubakar, I., Lipman, M., McHugh, T.D., Breuer, J. Direct Whole-Genome Sequencing of Sputum Accurately Identifies Drug-Resistant Mycobacterium tuberculosis Faster than MGIT Culture Sequencing. J Clin Microbiol 2018; 56(8): e00666-18.

33. Shockey AC, Dabney J, Pepperell CS. Effects of Host, Sample, and in vitro Culture on Genomic Diversity of Pathogenic Mycobacteria. Frontiers in Genetics 2019; 10.

34. Nimmo C, Shaw LP, Doyle R, et al. Whole genome sequencing Mycobacterium tuberculosis directly from sputum identifies more genetic diversity than sequencing from culture. BMC Genomics 2019; 20(1): 389. 
bioRxiv preprint doi: https://doi.org/10.1101/801308; this version posted October 31, 2019. The copyright holder for this preprint (which was not certified by peer review) is the author/funder, who has granted bioRxiv a license to display the preprint in perpetuity. It is made available under aCC-BY-NC-ND 4.0 International license.

RUNNING TITLE: Deep sequencing as a tool for TB control

483 35. Wyllie $\mathrm{D}$, Do $\mathrm{T}$, Myers $\mathrm{R}$, et al. M. tuberculosis microvariation is common and is 484 associated with transmission: analysis of three years prospective universal sequencing in 485 England. BioRvix 2019.

486 
bioRxiv preprint doi: https://doi.org/10.1101/801308; this version posted October 31, 2019. The copyright holder for this preprint (which was not certified by peer review) is the author/funder, who has granted bioRxiv a license to display the preprint in perpetuity. It is made available under aCC-BY-NC-ND 4.0 International license.

\section{RUNNING TITLE: Deep sequencing as a tool for TB control}

Figure 1. Transmission of M. tuberculosis in village K.

A.

Tree scale: $0.001 \longmapsto$

Sub-lineage, consistent with BAPS
$\square$ Mj-VA
$\square$ Mj-IIIA
$\square$ Mj-IIIB
$\square$ Mj-IIIC

B. CCATTCA CCATTCA CCATTCA $\times 10$ CCATTCA CCATTCA

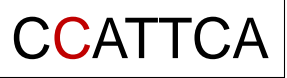

CCATTCA

CCATTCA

C.

CCATTCA

CCATTCA

CCATTCA

CCATTCA

CCATTCA

CCATTCA

CAATTCA

CAATTCA

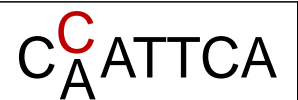

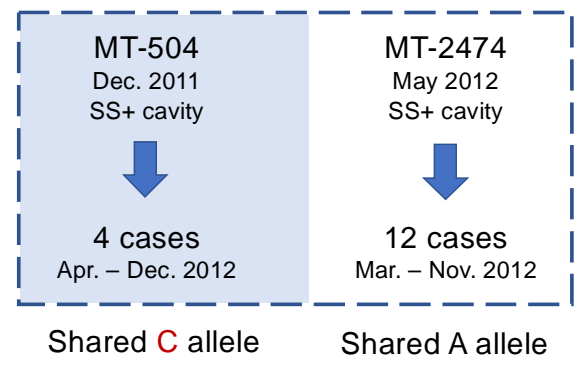

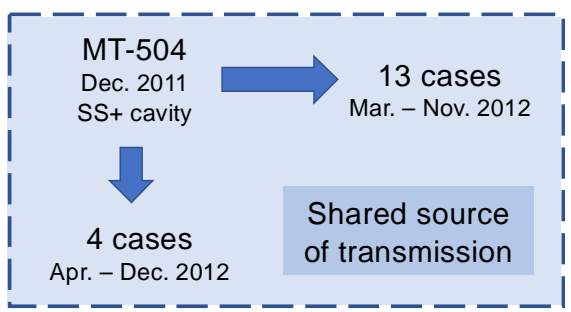

Panel A. Maximum likelihood tree of 62/65 cases diagnosed between 2007-2012 in village K based on consensus single nucleotide polymorphisms (cSNPs). After aligning to a local reference, MT-0080_PB, cSNPs were identified based on a minimum threshold of $\geq 95 \%$ of reads supporting the alternative allele. A core cSNP alignment was then produced with 103 positions. IQ-Tree (v.1.6.8 ${ }^{14}$ ) was then used to generate the tree using a KP3 model with correction for ascertainment bias. Model selection was based on the lowest Bayesian Information Criterion. Clusters were identified using hierarchical Bayesian Analysis of Population Structure. ${ }^{15}$ These clusters were consistent with the sub-lineages previously identified in ${ }^{3,4}$, thus only sub-lineage names are indicated. During this time period, there were two individuals who had a second episode of TB; stars are used to highlight these samples, with a different colour for each patient. MT-0080 is included in the alignment as the deep sequencing data from a sweep of all colonies identified a cSNP compared to the MT-0080_PB reference, which itself was generated from a single colony pick.

Panel B. Standard sequencing (to 40-50x), along with epidemiological data, had indicated that the Major [Mj]-IIIB sub-lineage was comprised of two subgroups of five and 13 patients, respectively. ${ }^{4}$ MT-504 was the suspected source case for the subgroup of five, which all shared a ' $\mathrm{C}$ ' allele at position 276,685 in H37Rv (position 276,544 in MT-0080_PB). In contrast, all members of the subgroup of 13 shared an 'A' at this position. Previously, MT-2474 was the suspected source case for this subgroup; this case was the first person with smear-positive (SS+) cavitary disease diagnosed in this subgroup.

Panel C. In contrast to standard sequencing, deep sequencing data revealed that, in fact, MT-504 - the presumed source for the subgroup of five cases and the first highly contagious case diagnosed in Mj-IIIB during the outbreak year - had both ' $\mathrm{C}$ ' and ' $\mathrm{A}$ ' alleles at this position (563:133 of reads, respectively), suggesting this was in fact the most probable source for both subgroups. 
RUNNING TITLE: Deep sequencing as a tool for TB control

514

515

516

517

\section{Tables}

Table 1. Comparison of alignments to H37Rv and MT-0080_PB

\begin{tabular}{|c|c|c|c|}
\hline & H37Rv (4,411,532 bp) & MT-0080_PB (4,426,525 bp) & P value \\
\hline \multicolumn{4}{|l|}{ Number of positions according to reference genome } \\
\hline Invariant reference across all samples, $\mathrm{n}(\%)$ & $4,018,786(91 \cdot 10 \%)$ & $4,084,195(92 \cdot 27 \%)$ & $<0.00005^{\mathrm{a}}$ \\
\hline Position was missing / low quality in at least one sample, $\mathrm{n}(\%)$ & $391,761(8 \cdot 88 \%)$ & $342,179(7 \cdot 73 \%)$ & $<0.00005^{\mathrm{a}}$ \\
\hline Position was an c/hSNP in at least one sample, $\mathrm{n}(\%)$ & $985(0 \cdot 22 \%)$ & $152(0 \cdot 00 \%)$ & $<0.00005^{\mathrm{a}}$ \\
\hline Shared cSNPs across all samples, $\mathrm{n}(\%)$ & $764(0 \cdot 02 \%)$ & $1(0 \cdot 00 \%)$ & $<0.00005^{\mathrm{a}}$ \\
\hline Shared hSNPs across all samples, $\mathrm{n}(\%)$ & $42(0 \cdot 00 \%)$ & $0(0 \%)$ & $<0.00005^{\mathrm{a}}$ \\
\hline \multicolumn{4}{|l|}{ Core pairwise distances } \\
\hline Core cSNPs vs. reference, median (range) & $791(790-792)$ & $3(1-65)$ & $<0.00005^{b}$ \\
\hline Core cSNPs between samples, median (range) & $3(0-64)$ & $3(0-66)$ & $<0.00005^{\mathrm{b}}$ \\
\hline
\end{tabular}

Legend. Based on these filters: Phred $<50$, Root Mean Square Mapping Quality (RMS-MQ) $\leq 30$, depth (DP) $<20$, Fisher Strand Bias (FS) $\geq 60$ and read position strand bias (ReadPos) <-8 and an allelic fraction of 95\% for cSNPs, with hSNPs classified when 5\% < ALT < 95\%. Quality metrics for the individual cSNPs/hSNPs identified in each sample are given in Table S2. ${ }^{a}$ Two sample test for difference in proportions. ${ }^{b}$ Wilcoxin Signed Rank test. 